\section{Regards sur l'économie allemande}

Bulletin économique du CIRAC

$91 \mid 2009$

Varia

\title{
60 ans de Loi fondamentale, 61 ans de Deutsche Mark
}

\section{Hans-Helmut Kotz}

Traducteur : Isabelle Bourgeois

\section{OpenEdition \\ Journals}

Édition électronique

URL : http://journals.openedition.org/rea/3700

DOI : $10.4000 /$ rea.3700

ISBN : 978-2-8218-0878-2

ISSN : 1965-0787

Éditeur

CIRAC

Édition imprimée

Date de publication : 1 mai 2009

Pagination : 31-44

ISSN : 1156-8992

\section{Référence électronique}

Hans-Helmut Kotz, « 60 ans de Loi fondamentale, 61 ans de Deutsche Mark », Regards sur l'économie allemande [En ligne], 91 | mai 2009, mis en ligne le 01 mai 2011, consulté le 04 mai 2019. URL : http:// journals.openedition.org/rea/3700 ; DOI : 10.4000/rea.3700 


\title{
60 ans de Loi fondamentale, 61 ans de Deutsche Mark
}

\author{
Hans-Helmut Kotz
}

"Le Deutsche Mark est soumis à des conditions institutionnelles qui limitent l'exercice de toute domination. Le DM aide à prévenir tout arbitraire. Cela en fait l'ami du citoyen et l'ennemi du pouvoir " (Ralf Dahrendorf, 1990, in Merkur, $n^{\circ} 497, p .580$ )

Certes, cela fait bien 10 ans et demi que le Deutsche Mark a disparu et qu'il a été remplacé par notre monnaie commune, l'Euro. Mais tout aussi indéniablement, il reste le symbole vivant de la reconquête de la prospérité des Allemands après les dévastations de la deuxième Guerre mondiale - pendant plus de quatre décennies d'abord pour les seuls Allemands de l'ouest avant de l'être aussi, pendant près d'une décennie, pour les Allemands de l'est. Beaucoup de penseurs ont interprété le Deutsche Mark comme un élément constitutif de la nouvelle identité allemande. D'autres, à l'opposé, voyaient dans cette identification avec la monnaie un succédané trop profane, un ersatz par trop commode en comparaison d'une idée plus noble de ce qui fait la quête de sens et le désir identitaire. Les débats sur cette question sont mus essentiellement par des approches normatives qui dépassent de loin l'objet de la politique monétaire au sens de l'approvisionnement approprié en monnaie ou de la fixation de taux adéquats. Car leur enjeu véritable est la confrontation des diverses approches de la définition du modèle ou de "l'ordre " économique et social désiré. Ces débats ne sont pas seulement internes à l'Allemagne, ils sont aussi franco-allemands - et nourrissent alors quelques " mésententes cordiales » entre nos deux pays.

\section{Le Deutsche Mark - bien plus qu'une monnaie?}

Face à l'ampleur de la thématique que représente l'évolution de l'économie de la République fédérale qui fête cette année son $60^{\circ}$ anniversaire et à laquelle est consacré ce numéro de Regards sur l'économie allemande, ma contribution se contentera nécessairement d'esquisser ici les enjeux monétaires sans évoquer les questions corollaires, relatives à l'Etat allemand ou à l'économie sociale de marché, présentées dans ce numéro par Alfred Grosser et Henrik Uterwedde. De même ne seront pas non plus évoqués ici les aspects plus symboliques dont l'importance est loin d'être négligeable, puisqu'ils marquent souvent de leur empreinte les débats publics. En guise exemple, on peut citer la question de savoir si la monnaie européenne devait porter le nom d'Ecu ou d'Euro ; elle venait en prolongement d'un débat qui s'était déroulé avant la création du Système monétaire européen et qui portait sur le sens à accorder à l'appellation ECU : était-ce un nom ou un acronyme? Un autre exemple de ces "symbolic uses of politics " est cette discussion qui a eu lieu en 1996 pendant quelques semaines en Allemagne et dont l'objet était de savoir s'il ne conviendrait pas d'accoler un suffixe à I'Euro, du type : Euro-DM, pour faciliter le transfert de l'aura du Deutsche Mark à la nouvelle monnaie et accroître ainsi sa réputation. Considérée sous un angle purement économique, la création de la monnaie européenne se présente sous des traits bien plus prosaïques : elle signifie la fin des taux de change nominaux en vigueur auparavant entre les Etats membres de la zone Euro. Ceux-ci ont désormais une politique monétaire unique, européanisée et un taux de change commun. Bien entendu, le taux de change réel (c'est-à-dire la relation entre les

ECU, Euro, Euro-DM...? L'importance des symboles 
Unions monétaires : points de cristallisation des divergences de vues prix des paniers de la ménagère dans ces Etats) perdure, entretenant ainsi en quelque sorte le maintien de l'Euro-DM ou de l'Euro-Franc.

Je m'en tiendrai donc ici à la seule monnaie (et partiellement à la politique financière), tout en plaçant mon analyse sous l'angle des influences réciproques et de la perception de ces diverses problématiques. Dans un premier temps, j'esquisserai dans ses grandes lignes l'évolution économique de l'Allemagne depuis 1948 , en centrant mon propos sur la variation du taux d'inflation et des parités monétaires (voir les graphiques ci-après) - donc sur la stabilité interne et externe. On pourrait à juste titre considérer une telle approche comme quelque peu réductrice, puisqu'elle n'aborde tout au plus qu'à la marge la problématique économique générale ou les questions de société. C'est donc la raison pour laquelle, dans cette analyse essentiellement chronologique, il sera mis l'accent, à propos des unions monétaires successives, sur des discussions de dimension francoallemande qui illustrent les divergences de vues, pas seulement économiques, constatées au sein de ce qu'il est convenu d'appeler, depuis les années 1960, le « couple franco-allemand». Des divergences semblables se laissant observer également au sein des débats nationaux respectifs, il paraît quelque peu simpliste d'opposer sans les nuancer les positions des Allemands et des Français, parfois présentées à l'évidence d'une manière caricaturale. II convient donc de garder à l'esprit que ces positions se résument à des opinions moyennes masquant la grande diversité des approches et plus encore l'existence de vues convergentes par-delà les frontières nationales. Sachant cela, j'exposerai ici les positions médianes respectives, c'est-à-dire les consensus dominants dans l'opinion.

\section{Préhistoire et genèse d'un symbole}

L'hyperinflation de 1922/23 avait fait le lit du nazisme

Misère et destruction de monnaie...

... appellent une nouvelle politique monétaire
La préhistoire de la Constitution monétaire octroyée aux Allemands par les Alliés Américains, Britanniques et Français - et donc 'leur' Deutsche Mark - est d'abord celle de l'échec du régime nazi. Celui-ci avait, en 1939, achevé de mettre au pas la Deutsche Reichsbank qu'il avait mise à contribution dès 1934 dans le financement de l'effort de guerre (Hansmeyer et Caesar, 1976). L'essor de l'idéologie nazie était la manifestation de l'érosion progressive de la République de Weimar. II était également nourri par le souvenir amer du chômage de masse et de l'hyperinflation de 1922/23. Celle-ci a été l'expérience clef qui avait transformé la société allemande et l'avait rendue littéralement « mûre pour Hitler » (« hitlerreif »), comme le formule l'écrivain Stefan Zweig dans son roman « Le Monde d'hier souvenirs d'un Européen » («Die Welt von Gestern »). Cette histoire, celle de la chute d'une monnaie allemande, s'était répétée ensuite moins d'un quart de siècle plus tard.

L'inflation retenue et camouflée sous le régime nazi (par le contrôle des salaires et des prix) a fini par aboutir, selon une logique implacable, à l'effondrement du Reichsmark. II vit, pendant trois ans, ses fonctions remplacées par des cigarettes. Le bon argent, rare (les cigarettes), supplanta le mauvais argent (le Reichsmark, dont la masse en circulation s'était multipliée par dix depuis le milieu des années 1930). La naissance du Deutsche Mark avait été déclenchée par la destruction de monnaie, précédée de trois années de terrible misère - une situation catastrophique au sens plein du terme. La création de richesse était tombée au quart de son niveau d'avant guerre. L'approvisionnement en denrées alimentaires était lamentable. " [Cette] paralysie de l'économie... faisait que la vie était à la limite du supportable et que l'humeur de la population oscillait entre une sourde léthargie et une révolte désespérée ; ... pendant la guerre même et immédiatement après, de nombreux experts de l'économie avaient perçu distinctement que par son financement 'discret' du réarmement et de l'effort de guerre, le gouvernement nazi avait généré un surplus monétaire impossible à réduire sans un profond assainissement de la monnaie » (Möller, 1976, p. 434).

La création du Deutsche Mark le 20 juin 1948 s'accompagna de la division par dix de la masse monétaire en circulation, d'une consolidation des dettes publiques et 
privées, et de la création d'une nouvelle banque, indépendante : la Bank deutscher Länder dans le but de garantir la stabilité de la valeur de la monnaie. En France, on espérait qu'ainsi serait désamorcée cette poudrière de l'Europe. Et très tôt, la politique monétaire de la Bank deutscher Länder s'attira en France des commentaires approbateurs saluant également son indépendance institutionnelle (voir par exemple Coulbois, 1957). Dans la Revue Economique, M. Mitzakis estimait en 1950 que la réforme monétaire « a été parfaitement conçue et adaptée à la nouvelle économie de l'ancien Reich, comme l'avait été en France, vingt ans auparavant, la réforme cartésienne du 25 juin 1926, qualifiée de 'miracle de Franc Poincaré' ». Et de fait, le miracle survint en Allemagne, le Deutsche Mark remplaça les cigarettes. Et du jour au lendemain, il y eut de nouveau des marchandises dans les magasins.

Sans conteste, l'expérience de l'effondrement de deux monnaies en l'espace d'une seule génération s'est profondément et sans soute durablement ancrée dans la mémoire collective (James, 1998). Quoi qu'il en soit, l'inflation : c'est-àdire la perte durable du pouvoir d'achat, n'a jamais été considérée en Allemagne ni encore moins acceptée par la société allemande comme ce « social mollifier » (Bronfenbrenner), ce « lénifiant social » qu'y voient d'autres sociétés (Pierenkemper, 1996). Et on reproche toujours au chancelier Helmut Schmidt d'avoir affirmé, lors d'une campagne électorale au début des années 1970 qu'il préférait « un taux d'inflation de $5 \%$ à un taux de chômage de $5 \%$ ».
Une aversion profonde contre l'inflation...

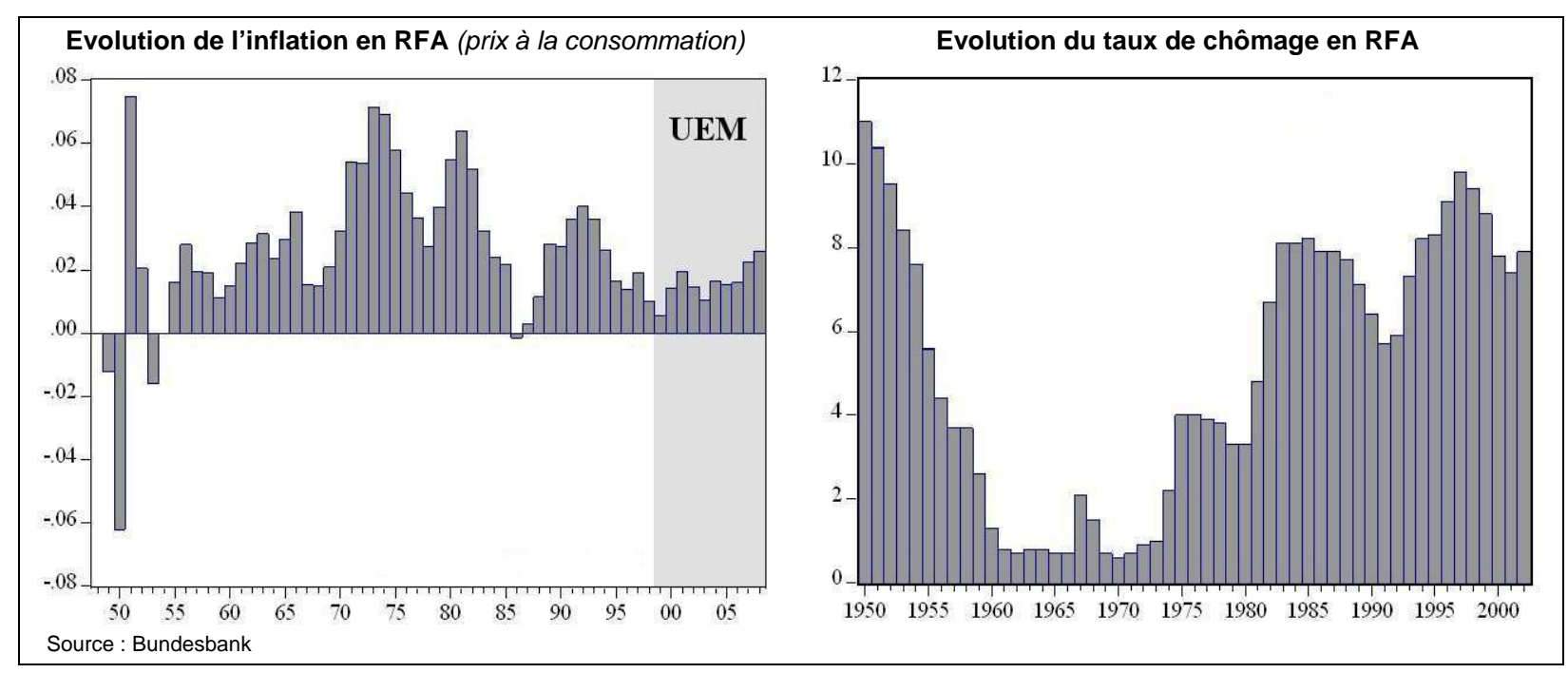

Si l'aversion qu'inspire aux Allemands l'inflation - et, en toute logique, le corollaire qu'elle trouve dans leur prédilection pour la stabilité - frise aujourd'hui le stéréotype, celui-ci est pourtant loin d'être dénué de tout fondement. Car cette aversion ... qui conditionne attentes et anticipations ne semble pas sans conséquences pour ce qui est de la formation de l'opinion publique. Deux économistes allemands enseignant aux Etats-Unis, Ulrike Malmendier et Stefan Nagel, viennent de démontrer magistralement dans un article publié en 2009 comment les expériences collectives de l'inflation influent sur la formation des attentes (l'anticipation) dans l'espace public. Ils expliquent ce comportement en se fondant sur le concept du processus adaptatif d'apprentissage pour développer un indicateur permettant de prendre en compte l'expérience de l'inflation recueillie tout au long de la vie. La notion ancienne d'anticipation, aujourd'hui passée de mode dans les sciences économiques, repose sur l'idée que les individus ne cessent de commettre des erreurs d'appréciation sur l'évolution de l'inflation, mais qu'ils n'en tirent pas les enseignements : ils n'agissent donc pas de manière totalement rationnelle. Or cette approche entre en contradiction avec les critères de congruence qu'on exige généralement des modèles économiques. Pourtant, et cela ne surprendra guère les non-économistes, l'expérience vécue conditionne la formation des anticipations. Et celles-ci déterminent les 
La Bundesbank, vue comme la gardienne du pouvoir d'achat

Les conflits d'approches sur l'indépendance des banques centrales..

... portent moins sur les moyens que sur les objectifs

C'est la confiance de l'opinion qui fonde l'indépendance revendications en matière de prix ou de salaires. L'anticipation joue donc un rôle majeur dans la formulation des politiques.

Cette aversion fondamentale contre l'inflation a surtout constitué pour la Bundesbank et la Bank deutscher Länder qui la préfigurait un capital qu'elles ont su faire fructifier. La Bundesbank pouvait se reposer sur une opinion publique que l'amère expérience de l'inflation avait conduite à conférer une haute valeur à la culture de la stabilité et qui dès lors considérait la Buba comme la gardienne de son pouvoir d'achat. Et Adam Posen (1996) démontre que c'est justement ce large appui qui explique pourquoi l'Allemagne a connu depuis le début des années 1950 une inflation modérée en comparaison des autres pays - et ce, pardelà même l'indépendance de la Bundesbank qui en est la raison complémentaire. Ceci est un argument que Karl-Otto Pöhl, ancien président de la Bundesbank, ne cessait lui aussi de rappeler (voir à ce sujet Marsh, 1992).

Cela nous amène au premier exemple de cette dissonance culturelle qu'on observe parfois dans les opinions de nos deux pays. II s'est manifesté au cours d'un débat télévisé diffusé en France en 1992 peu avant le referendum sur le Traité de Maastricht et où s'opposaient Philippe Séguin, incarnation du « non », et François Mitterrand. Un point que Monsieur Séguin considérait comme inacceptable, opinion qu'il savait à l'évidence partagée par une large partie des téléspectateurs, était le fait que soit requise dans ce cadre l'autonomie de la Banque de France. Cette exigence lui semblait incompatible avec la conception traditionnelle du rôle de l'Etat (c'était d'ailleurs là, au début des années 1990, la vision internationale standard, qui prédominait notamment en Angleterre sous l'égide de sa première ministre Thatcher). Quant au Président Mitterrand, il se contenta d'écarter cette objection d'un geste condescendant de la main, manifestant par là qu'il ne fallait pas prendre tant au sérieux la question somme toute assez théorique de l'indépendance de la banque centrale européenne.

Tout respect gardé, une telle présomption ne pouvait qu'engendrer quelque frustration. Les banques centrales européennes prennent leur indépendance réellement très au sérieux. Les lecteurs familiarisés avec les termes des débats internes à l'Allemagne savent l'importance qu'on accorde à la différence entre la notion d'indépendance qui ne laisse place à aucune ambiguïté et celle d'autonomie qui laisse une trop grande marge d'interprétation. Et de fait, la garantie de l'indépendance de la Banque Centrale Européenne dont la moindre modification des statuts ne pourrait s'opérer que par referendum est bien plus forte que celle de la Bundesbank qui peut être invalidée par une simple procédure législative au Bundestag. Le gouvernement fédéral ne s'était d'ailleurs pas privé de brandir cette menace à plusieurs reprises dans les années qui avaient suivi sa création, c'est-à-dire dans les années 1950... De plus, la BCE est dotée de l'indépendance tant en ce qui concerne les moyens que les objectifs de sa politique. Elle dispose donc d'une autonomie pleine et entière non seulement pour fixer ses taux et pour définir l'usage qu'elle fait des outils dont elle dispose pour assurer les liquidités - ceux-ci étant d'une importance stratégique en ces temps de crise financière -, mais aussi dans la réalisation de son objectif qui est de contenir l'inflation à « moins de $2 \%$, mais près de $2 \%$ ». Cet objectif, qui constitue le cœur des missions de la BCE, ressemble beaucoup à la «Preisnorm » : cette « norme des prix », objectif que s'était fixé, en pleine autonomie elle aussi, la Bundesbank. Une telle indépendance semble d'ailleurs disproportionnée à la plupart des observateurs anglo-saxons lesquels, à l'instar de Stanley Fisher et Guy Debelle qui sont les auteurs de cette distinction, accordent à la banque centrale certes l'indépendance des moyens, mais pas celle des objectifs.

Rappelons ici que, même en Allemagne, l'indépendance de la Bundesbank n'a pas toujours fait l'unanimité. L'exemple le plus célèbre en est un discours tenu par le chancelier Adenauer à Gürzenich le 23 mai 1956 et dans lequel il critiquait vertement la Bank deutscher Länder (préfiguration de la Bundesbank) pour avoir une nouvelle fois resserré ses taux en dépit d'innombrables interventions politiques. Ce discours s'insérait dans le contexte de l'élaboration de la Loi sur la 
Bundesbank et intervenait après que la Bank deutscher Länder avait d'une part relevé d'un point de pourcentage son taux d'escompte et, d'autre part, réduit ses quotas de réescompte, restreignant ainsi les possibilités ouvertes aux banques de recourir au crédit. Cette mesure de politique monétaire était, selon Adenauer, " un couperet tombant sur les petites gens et qui de ce fait m'attriste beaucoup ". Or l'opinion publique ou, pour être plus précis : l'opinion médiane des leaders d'opinion, s'opposa au chancelier. Et c'est ainsi que le « discours de Gürzenich » du chancelier Adenauer se transforma a contrario en l'une des pierres angulaires portant les fondations d'une Bundesbank indépendante (pour plus de détails, voir Neumann, 1998). D'autres charges contre son indépendance auraient eu lieu en coulisses du temps du gouvernement Schmidt. Mais de telles menaces sont toujours restées sans conséquence, la grande confiance que portait l'opinion publique à la Bundesbank étant l'un des piliers de son autonomie. Aujourd'hui, c'est dans seize opinions publiques qu'il revient au Système européen des banques centrales de développer cette position.

\section{Stabilité - la dimension européenne}

Mais jusqu'à l'effondrement du système de parités fixes de Bretton-Woods, c'est toutefois dans un autre contexte que l'autonomie des choix de politique monétaire de la Bundesbank était mise en cause. Tant que les Etats-Unis menaient leur politique de stabilisation de la valeur monétaire du dollar, nul ne considérait comme problématique l'obligation faite à la Bundesbank d'intervenir pour maintenir le niveau des cours du Deutsche Mark par rapport au Dollar. II n'y avait pas de conflit entre objectifs externe et interne de politique monétaire durant les années 1950, marquées par le «Dollar fort 》. Mais au cours de cette décennie commençait à s'esquisser la tendance à un Mark fort qui déboucha, dès le milieu des années 1950, sur la première discussion sur une éventuelle réévaluation du Deutsche Mark. La majorité du Conseil de la banque centrale était opposée à la réévaluation finalement intervenue en mars 1961. Et quand en 1968, dans le contexte des turbulences que subissait le Franc, fut envisagée une nouvelle réévaluation, une nette majorité se prononça pour. Et pourtant, la Bundesbank souhaitait en réalité préserver le système de parités fixes de Bretton-Woods. Mais, comme le révèle Manfred Neumann, le hasard de l'histoire en a décidé autrement - ou plutôt le congé maladie à la fois du ministre fédéral des Finances, Helmut Schmidt, et du président de la Bundesbank, Karl Klasen, que son remplaçant provisoire, le vice-président de la Bundesbank, Otmar Emminger, mit à profit pour persuader le chancelier Willy Brandt de fermer les marchés des changes et ainsi sortir du système. Karl Schiller, le prédécesseur de Helmut Schmidt au poste de ministre fédéral des Finances, plaidait depuis deux ans déjà pour un flottement encadré des parités ; la majorité du Conseil central de la Bundesbank y était opposée. "Depuis la fin du système monétaire de Bretton-Woods, il ne s'est plus jamais produit qu'une majorité se dégage au sein du Conseil central pour céder à l'illusion que la Bundesbank devait préserver à tout prix les parités monétaires tout en accomplissant dans le même temps sa mission de garantir la valeur interne de la monnaie " (Neumann, 1998, p. 337 sq.).

La perte de «l'ancre nominale » qu'était la parité des monnaies, pour employer la métaphore en cours depuis quelque temps, exigeait un substitut. II fut trouvé dans la focalisation sur l'accroissement de la masse monétaire - conformément à cette sentence de Milton Friedman selon laquelle " l'inflation est à long terme, toujours et partout, un phénomène monétaire ». La mise en œuvre du changement d'orientation fut précédée d'un débat, déclenché à la fin des années 1960 par Rolf Gocht, un des membres du directoire de la Bundesbank, qui en avait lancé l'idée sous forme de "pensées iconoclastes nocturnes ». Parmi les participants à ce débat figuraient également de jeunes économistes de la Bundesbank comme Manfred Neumann et Wolfgang Gebauer, de même que l'économiste américain Jerry Jordan qui faisait un stage à Francfort à cette époque.
La fin du système de parités fixes de Bretton-Woods amène...

... une focalisation sur l'évolution de la masse monétaire 
Critique du « monétarisme pragmatique » de la Buba

Le SME d'abord controversé..

... pour l'asymétrie des parités
Cette nouvelle approche théorique axée sur l'évolution de la masse monétaire et dont la forme concrète a subi des évolutions au fil du temps - elle se concentrait d'abord sur la seule masse monétaire-banque centrale (liquidités, dettes domestiques des banques pondérées des taux de réserves obligatoires) avant de prendre pour référence, à partir de 1987, la masse monétaire M3 (liquidités, dépôts à vue et dépôts à terme) - n'était toutefois en rien l'application directe de la pensée de Friedman. Car la possibilité de choisir l'agrégat monétaire à piloter et, surtout, sa latitude dans la détermination des objectifs, laissaient à la Bundesbank une grande marge d'appréciation. Dans sa lecture critique d'un article de Helmut Schlesinger où celui-ci esquissait la conception de la politique monétaire de la Bundesbank, l'économiste américain Alan Blinder (1987) y vit la parfaite description d'une politique agissant au cas par cas, donc d'une politique discrétionnaire et non pas d'une politique obéissant à des règles. Et de fait, son « monétarisme pragmatique » (Schlesinger) permettait à la Bundesbank la moitié du temps - et surtout sans tenir compte du fait qu'elle avait à plusieurs reprises manqué ses objectifs sans les corriger (ce qu'il est convenu d'appeler «basisdrift ») - de ne pas respecter ses obligations quant à l'objectif intermédiaire.

C'est sur cette toile de fond de politique monétaire que fut considérée l'intégration dans le Système monétaire européen (SME) lancé par Helmut Schmidt et Valéry Giscard d'Estaing. Celui-ci avait été conçu et élaboré dans ses grandes lignes pour le Sommet de Brême en juillet 1978 par Horst Schulmann et Bernard Clappier, ce dernier ayant auparavant "coopéré à l'élaboration du Plan Schuman (autrement dit, il était dans la mouvance de Jean Monnet) » (Schmidt, 1990, p. 254). Du fait des obligations d'intervention que créait cette intégration, elle rendait potentiellement problématique l'objectif prioritaire du maintien de la valeur de la monnaie. Helmut Schmidt rappelle que "l'idée [du SME est] née au cours d'un entretien entre nous deux [Schmidt et Giscard d'Estaing]. Peut-être était-elle dans l'air, comme on dit, car il y avait à l'époque un débat semi-public sur l'avenir monétaire de l'Europe auquel Otmar Emminger (président de la Bundesbank) apportait une contribution négative et Roy Jenkins (président de la Commission de la CE), une contribution positive" (Schmidt, 1990, p. 249). Le passage intitulé «Nous créons un Système monétaire européen" (p. 247-272, dans le chapitre «Bonne Entente avec la France ») où il évoque ce souvenir a entre autres ceci de remarquable qu'il permet de comprendre pourquoi Helmut Schmidt avait lancé la réalisation de ce projet hors des procédures habituelles et sous le sceau du plus grand secret: " conformément à ce qui était convenu, nous avons laissé dans l'ignorance nos bureaucraties nationales, de même d'ailleurs que la bureaucratie de Bruxelles (seul Roy Jenkins était informé) ».

Le SME, devenu de plus en plus contraignant au fil du temps, avait bien mené sur une longue période à une "zone de stabilité monétaire », mais du fait de son orientation non-symétrique à la grille des parités bilatérales - une architecture qui ne trouva jamais pleinement l'assentiment de la France -, il se traduisit par une forte désinflation au sein de l'Europe. Selon le Plan Fourcade de 1974, par exemple (Fourcade était alors ministre de l'Economie et des Finances), cette asymétrie devait être réduite d'une part grâce à son orientation sur la European Unit of Account et non plus sur des parités bilatérales, d'autre part grâce à une hausse des lignes de crédit et une défense des parités à l'intérieur des corridors. II s'agissait de redistribuer les charges de réajustement entre pays déficitaires et pays excédentaires. Cette asymétrie ne correspondait pas non plus aux conceptions du chancelier Schmidt. Pourtant elle était quasi incontournable, car obéissant à une logique décrite au début des années 1960 par Robert Mundell sous la forme du triangle funeste qui veut qu'une politique monétaire autonome, la libre circulation des capitaux et des taux de change fixes ne soient pas toujours compatibles entre eux. Le maintien du taux de change liait donc de plus en plus étroitement les politiques monétaires nationales au Deutsche Mark - monnaie considérée comme la plus forte sur le marché. 
Elle devint donc la monnaie d'ancrage, ce rôle traduisant la performance de l'économie allemande (sous l'angle de l'inflation, de la balance des paiements, de l'évolution de la productivité, etc.) par comparaison avec celle des autres pays du SME (Dyson, 1994, p. 16 ; Giersch, 1994, p. 253). Or c'est justement cet arrimage - le fait que chacun se lie les mains de son propre chef - qui était censé rendre le processus de désinflation (politiquement) moins coûteux pour les pays partenaires. Un système plus symétrique n'aurait pas mené à la stabilité recherchée, celle-ci étant la conséquence directe de l'action du marché. La crainte d'une perte de prestige inhérente à une dévaluation finit par forcer l'alignement politique. Malgré tout, comme le montrent les nombreux réajustements, le SME donna lieu à une application plus souple que le système de BrettonWoods. Mais le SME créa aussi les conditions pour que l'idée "un marché, une monnaie » (devise de la Commission européenne pour le passage à l'Euro) devienne de plus en plus plausible. En particulier lorsque la France, après 1983, eut opéré son revirement vers la politique du Franc fort puis, ensuite, de la désinflation compétitive.

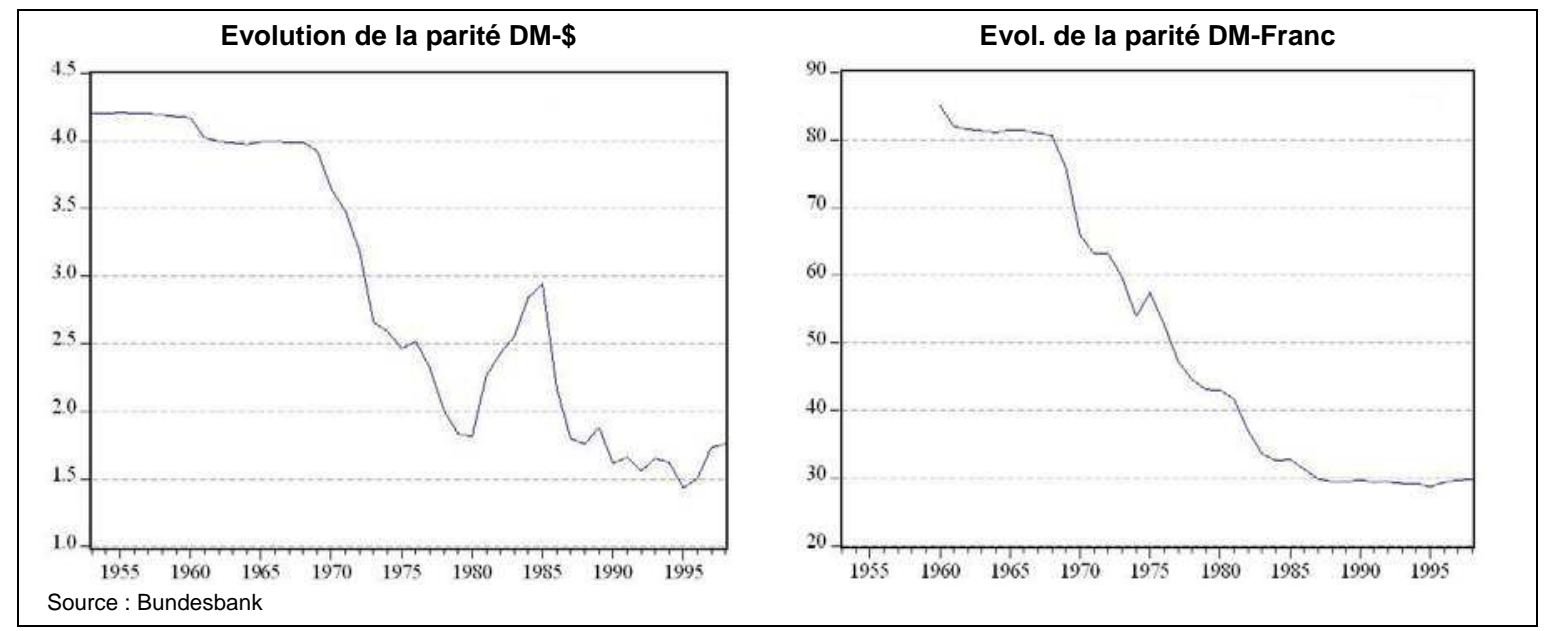

Après le deuxième choc pétrolier au début des années 1980, la Bundesbank n'avait certes pas laissé l'inflation atteindre en Allemagne le niveau connu par d'autres pays (ce qui avait généré au sein du SME de fortes tensions qui purent être calmées grâce à l'ajustement des parités). Mais elle ne poursuivait pas non plus l'objectif de ramener brutalement l'inflation à sa norme d'environ $2 \%$, préférant prendre pour référence le possible, c'est-à-dire s'accommodant d'une inéluctable hausse des prix. Cela revenait implicitement à considérer qu'une politique désinflationniste trop rigoureuse aurait impliqué un renoncement disproportionné à ses objectifs de stabilisation de l'output. Elle poursuivait donc une politique de «monétarisme pragmatique ». Cela s'exprima en particulier dans les estimations selon lesquelles ce qu'on appelait à l'époque les «fonctions de réaction de la banque centrale », lesquelles tentaient d'appliquer la "politique des taux » en la mettant en relation avec l'évolution de l'inflation, de la croissance et des taux de change, montrèrent que dans le cas de la Bundesbank, celle-ci accordait à peu près autant de poids à l'évolution de l'inflation qu'à celle de la croissance du PIB (Kotz, 1992). Dans les années 1950 déjà, on pouvait constater que la banque centrale allemande tenait compte dans ses décisions des «impératifs de politique industrielle »-c'est-à-dire de ce qu'on appellerait aujourd'hui le développement du potentiel comme de la création d'un excédent de valeur -, essentiellement sous l'angle de leur possible effet de soutien aux exportations (Holtfrerich, 1998 ; Giersch et al., 1992).

Qu'il nous soit permis ici de faire une petite entorse à la stricte chronologie. En septembre 1992, la Grande Bretagne se vit contrainte de quitter le Système monétaire européen (voir, sur cet épisode particulièrement éprouvant aussi sous l'angle de la diplomatie monétaire, Marsh, 2009). Elle se vit ainsi privée opte elle aussi pour un pilotage axé sur l'évolution de l'inflation 
elle aussi de son ancrage monétaire. La Bank of England décida alors rapidement de réorienter sa politique en abandonnant sa référence à l'objectif intermédiaire (par exemple la masse monétaire) au profit d'un pilotage axé sur l'objectif final : le contrôle direct de l'évolution des prix à la consommation. II est d'ailleurs intéressant de rappeler à cet égard que l'actuel président de la banque centrale américaine, Ben Bernanke, qui défendait autrefois, en tant que scientifique, l'approche du pilotage par l'inflation, compte la Bundesbank parmi les membres implicites et inavoués de ce club. Andrew Brociner et Odile Chagny parviennent à la même conclusion : "La Bundesbank privilégie plutôt une approche pragmatique, selon laquelle le respect de l'objectif final de préservation de la valeur de la monnaie que lui assignent ses statuts prime sur l'interprétation dogmatique de la théorie quantitative de la monnaie ».

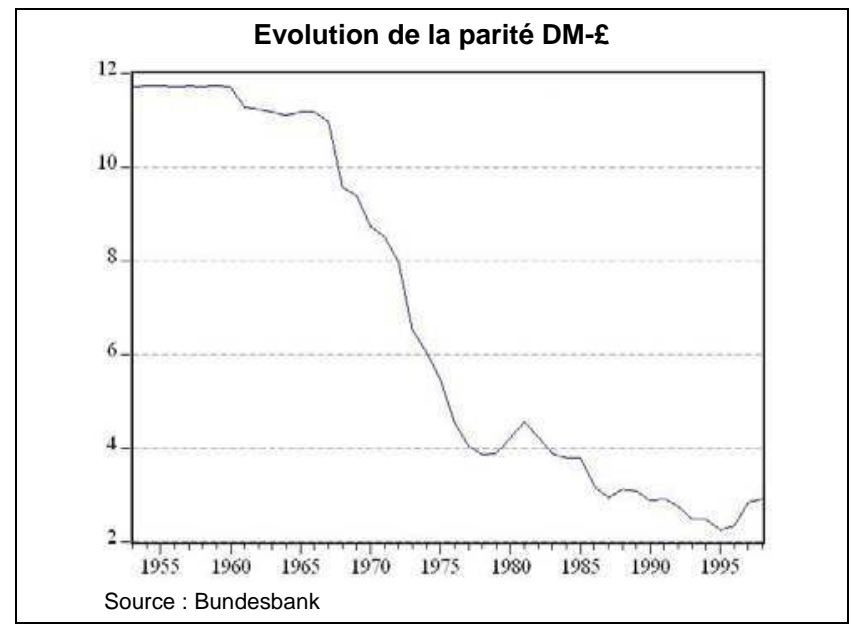

Ce point est important pour comprendre la perception de l'opinion publique, en tout cas celle de l'opinion médiane : ce qui importe réellement, c'est que l'objectif final soit atteint. Et c'est justement sur cela que reposait la haute réputation de la Bundesbank : l'opinion la percevait comme la gardienne du Deutsche Mark, autrement dit, de la stabilité du pouvoir d'achat. Soit dit en passant, c'est là aussi la raison pour laquelle Erich Schneider, dont la pensée marquait les sciences économiques allemandes dans les années 1960, plaidait pour une approche orientée sur l'objectif final.

\section{Union monétaire et problèmes de compréhension}

Mésentente franco-allemande sur l'union monétaire intra-allemande

Coût prohibitif de l'Unité ?
C'est surtout au cours de deux périodes, toutes deux liées à une union monétaire, que s'observent les plus franches « mésententes » franco-allemandes. La première concerne l'union monétaire intra-allemande de 1990, c'est-à-dire l'introduction du Deutsche Mark dans les nouveaux Länder qui succéderont à l'exRDA. Le « démarrage à froid » imposé à l'économie est-allemande (Gerlinde et Hans-Werner Sinn, 1991) s'accompagnait d'un potentiel inflationniste que la Bundesbank se devait de juguler si elle ne voulait pas risquer de faillir à sa mission. Mais, du fait des restrictions imposées par le SME, sa politique eut pour conséquence une hausse des taux dans les Etats partenaires, ce qui ne pouvait que nourrir de sérieuses tensions entre ceux-ci. A la hausse progressive des taux s'ajouta celle de la prime aux réajustements de parités, ce qui renforça encore les tensions et déclencha en 1992 et 1993 la crise du SME. La seule solution, à savoir une réévaluation unilatérale du Deutsche Mark, n'était pas envisageable, pour des raisons politiques.

Plus révélateur encore est un débat qui s'était fait jour avant cette crise. L'économiste français Serge-Christophe Kolm avait publié dans Le Monde une tribune libre d'une pleine page (en page 2, si mes souvenirs sont bons) et dans laquelle il déclarait sa grande hostilité à I'Unité allemande, considérant celle-ci 
comme un pari risqué au coût potentiellement prohibitif. Or cette vision « teutomaniaque » à outrance, alors très répandue, avait trouvé sa promotrice assurément la plus ardente en la personne de la première ministre britannique Margaret Thatcher. Les écrits de nombreux observateurs tout comme les souvenirs des principaux acteurs impliqués révèlent combien, à l'époque, le projet de I'Unité était controversé (Attali, 1996). Mais ils font apparaître aussi que l'opportunité pour la mener à bien ne se présentait tout au plus que pendant un très bref laps de temps (Winckler, 2002, p. 525). C'est assurément ce constat qui détermina le chancelier Kohl à présenter au Bundestag le 28 novembre 1989, soit moins d'un mois seulement après la chute du Mur, son « Programme en dix points » pour l'Unité allemande - un programme décidé en l'absence de toute coordination. Or ce programme se heurta à la farouche opposition non seulement de Gorbatchev, mais aussi d'un certain nombre de partenaires européens, tout particulièrement de Margaret Thatcher ou de son homologue italien Giulio Andreotti.

Cette opposition est directement liée à un aspect très contesté : la relation présumée indissociable entre Unité allemande et abandon du Deutsche Mark qui, tant pour les parties prenantes que pour les observateurs participants, semble aussi bien établie que démentie. La logique de l'Histoire s'éclaire toujours a posteriori. Le monde des possibles semble toujours plus vaste ex ante et reste souvent controversé même rétrospectivement. Néanmoins, les publications de nombreux acteurs impliqués permettent maintenant d'accréditer une lecture en termes d'échange négocié. Or l'initiateur en fut, entre autres selon Dyson et Featherstone (1999), “ l'entrepreneur politique » Hans-Dietrich Genscher, alors ministre fédéral des Affaires étrangères, qui aurait souhaité troquer la souveraineté monétaire contre une influence politique accrue. Et lors du Sommet de Hanovre en juin 1988, celui-là même où le Comité Delors fut investi de sa mission, le chancelier Kohl prit le parti de défendre le projet d'une monnaie unique - à un moment où rien ne laissait présager encore l'Unité allemande. Le ministère allemand des Finances, quant à lui, était pour le moins défavorable à ce projet. Quoi qu'il en soit, et indépendamment de cette genèse controversée, ce qui importe dorénavant, c'est que dix ans après sa création, I'Union monétaire ait rempli sa véritable mission : la garantie d'un faible taux d'inflation au sein de la zone Euro. Quant à la question de savoir comment se présenterait une Europe sans monnaie unique dans le contexte de la crise actuelle des marchés financiers, elle semble quelque peu rhétorique. Le Président Mitterrand en tout cas avait, avant le Sommet des chefs d'Etat et de gouvernement qui s'était tenu à Strasbourg les 8 et 9 décembre 1989, obtenu du chancelier Kohl l'assurance que la République fédérale respecterait largement la vision française d'une Union économique et monétaire européenne. II se trouve bien sûr aussi des voix pour soutenir sans ambages qu'il se serait agi d'un échange donnant-donnant : Unité allemande contre abandon du Deutsche Mark (voir à ce sujet Dyson et Featherstone, 1999, ou encore Aeschimann et Riché, 1996). Une telle approche n'est en tout cas pas sans une certaine congruence historique, puisque la partition de fait de l'Allemagne avait aussi pour origine la création du Deutsche Mark en 1948...

Mais l'Unité allemande - du moins en ce qui concerne ses modalités - ne faisait pas l'unanimité en Allemagne non plus. Ainsi, par exemple, le philosophe très reconnu Jürgen Habermas assimilait l'unification à " un chauvinisme du Deutsche Mark propre aux gens bien nourris ». II plaidait avec ardeur pour organiser le rattachement de la RDA à la RFA par voie de referendum. Sans détailler ici la doctrine assurément ambitieuse de Habermas car elle repose sur son concept d'une « situation discursive idéale », il n'est pas moins permis d'affirmer que, dans la situation politique brièvement esquissée plus haut, l'approche du philosophe n'était guère appropriée. L'enjeu des débats était la question de savoir si l'unification se ferait en vertu de l'article 23 de la Loi fondamentale (procédure d'adhésion) ou en vertu de la pleine liberté de décision sur
Unité allemande contre abandon du Deutsche Mark ?

En RFA, controverse sur les modalités politiques de I'Unité 
Les Allemands de l'est appelaient de leurs vœux le DM

Une parité progressive DM-Mark est était irréaliste une Constitution conférée au peuple par l'article 146, par exemple par voie de referendum. En effet, si la Loi fondamentale a depuis son adoption un caractère explicitement provisoire, c'est qu'elle était à l'origine «la Constitution d'un Etat partiel », d'où son nom (Vorländer, 2009). Vue sous cet angle, la position défendue par Habermas était bien sûr parfaitement légitime. Et elle est à l'origine de la genèse d'une notion digne de réflexion dans une approche européenne: celle de patriotisme constitutionnel postnational (Habermas avait prôné une approche comparable pour l'Europe). Mais étant donné le contexte historique et économique après la chute du Mur, procéder par referendum n'aurait vraisemblablement pas mené à l'Unité allemande (ainsi l'analyse tranchée livrée par Winckler, 2002).

Cette position négligeait totalement, en effet, la situation économique désastreuse d'une RDA qui, après avoir trop longtemps puisé dans sa substance, se trouvait alors au bord de l'implosion. Les conditions de vie en Allemagne de l'Est, telles que les a décrites par exemple de manière très vivante l'historien américain Jonathan Zatlin, expliquent à quel point le slogan «Si le Deutsche Mark ne vient pas à nous, nous irons à lui » («Kommt die D-Mark nicht zu uns, kommen wir zu ihr ») résume fidèlement ce que ressentaient alors les citoyens des futurs nouveaux Länder. Une large majorité d'entre eux appelaient de leurs vœux l'adhésion de la RDA au " territoire où s'applique la Loi fondamentale ", conformément à son article 23 - mais de préférence sans avoir à en payer le prix (Winckler, 2002).

Dans ces conditions, l'idée de piloter l'intégration des deux économies allemandes via une modification progressive de la parité entre le Deutsche Mark et le Mark de la RDA, comme l'avait par exemple soumise au gouvernement dans une lettre le Conseil des Sages, n'était guère applicable. Car les Allemands avaient la possibilité de comparer au concret le pouvoir d'achat que leur apportait leur travail à l'Est ou à l'Ouest. Et cette possibilité constituait une d'autant plus vive incitation à la migration que les perspectives d'emploi locales n'étaient guère réjouissantes.

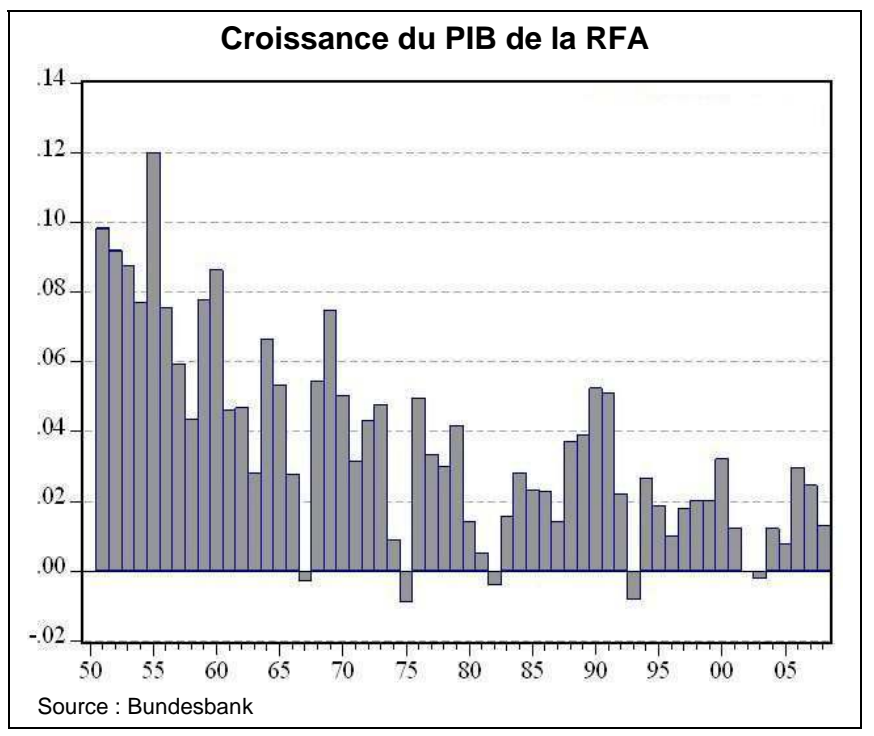

Le rattrapage rapide de l'ex-RDA, une illusion
Quant à la vision de "paysages fleuris » (Helmut Kohl) et d'un rattrapage en l'espace de 5 ans, elle ne pouvait d'emblée que tenir du rêve. Compte tenu des revenus par tête respectifs et en présupposant que la dynamique de croissance à moyen terme de l'Allemagne de l'ouest se maintienne à niveau constant, un délai de 5 ans n'eût été réaliste qu'à la condition que les économies des nouveaux Länder connaissent un taux de croissance annuel de $25 \%$. Car même avec une croissance comparable à celle des années du «miracle économique », il aurait fallu près de 15 ans pour que le rattrapage se fasse (Kotz, 1990). 
Quoi qu'il en soit, le basculement au Deutsche Mark et les taux de change alors adoptés (pour les transactions courantes, c'est-à-dire les salaires, les loyers, etc., ils étaient de 1 DM pour 1 Mark est ; pour les avoirs, de 1 pour 1,8) furent pour l'économie de l'ex-RDA un choc qu'elle mit très longtemps à surmonter. II faut dire aussi qu'au moment de l'Unité, on se faisait encore, à l'Ouest, des illusions quant au potentiel économique de l'ex-RDA. Une remarquable étude publiée en 1991 par George Akerlof, Andrew Rose, Janet Yellen et Helga Hessenius, avait établi, en s'appuyant sur les données du Commissariat au Plan de la RDA, qu'à peine $8 \%$ des entreprises y étaient à même de couvrir leurs coûts variables sur la base des prix du marché mondial. Au vu de ce constat, l'ampleur réelle du travail de restructuration saute aux yeux- et on comprend mieux pourquoi les évaluations que faisait la Treuhandanstalt de la valeur du patrimoine qu'elle avait à gérer ne cessaient de baisser jusqu'à ce qu'il ne lui reste plus, à la fin, qu'à constater une énorme perte nette.

L'économiste américain Robert Barro avait calculé, en partant de constats empiriques, qu'il faudrait plus d'une génération pour que l'économie est-allemande parvienne à mi-chemin du processus de transition. Certes, en ce qui concerne les revenus nets par tête, qui atteignent désormais $90 \%$ du niveau moyen de l'Allemagne de l'ouest, le rattrapage s'est fait beaucoup plus vite que prévu. Mais la création de richesse est restée nettement en dessous du niveau de la consommation, ce qui creusa un énorme déficit structurel dans la balance des paiements courants. On tenta longtemps de le réduire grâce à d'importantes subventions dont le montant s'élevait, au début, à environ $6 \%$ du PIB ouestallemand. Et comme il était politiquement inconcevable que la transition s'effectue grâce à un différentiel salarial, la conséquence en fut un phénoménal mouvement migratoire - des régions est-allemandes à faible dynamisme structurel vers les zones prospères à forte concentration d'activité à l'ouest. Aujourd'hui encore, quelque $3 \%$ du PIB ouest-allemand sont transférés en Allemagne de l'est pour y financer le déficit courant.

Comme elle s'accompagnait d'une hausse des taux (voir supra), l'Unité fut un choc d'une grande ampleur pour le Système monétaire européen. Pour préserver les taux de change au sein d'une grille de parités où prédominait le Deutsche Mark, les banques centrales des partenaires de l'Allemagne au sein du SME durent harmoniser leurs taux. II s'ensuivit souvent des taux d'intérêt réels en contradiction totale avec les impératifs économiques domestiques. La pression augmenta à tel point jusqu'à la fin de l'été 1992 que la Grande Bretagne fut contrainte de quitter le SME le 16 septembre. La Lire italienne fut dévaluée d'un quart, et les monnaies espagnole, portugaise et suédoise connurent de fortes tensions.

Le conflit entre les objectifs économiques domestiques et la préservation de la parité dans le SME, que les marchés suivaient de très près, perdura jusqu'au cours de l'été 1993, entretenu par un ralentissement économique généralisé. En juin 1993, le Nouvel Observateur publia un article intitulé : " Et si on changeait tout ? ». II relatait le mécontentement qu'éprouvaient "parlementaires et grands patrons face à une garde prétorienne du Trésor » qui avait un œil rivé sur la défense du Franc et l'autre, sur la préservation des grands équilibres («Bercy brûle-t-il ? »). Dans un entretien paru dans le même numéro du Nouvel Observateur, Olivier Blanchard affirmait que seule une sortie du SME permettrait à la France de renouer avec la croissance, puisqu'ainsi les taux pourraient être abaissés à un niveau favorable à la relance de la demande intérieure. Ce n'est que lorsque l'élargissement des marges de fluctuation à $15 \%$ fut choisie comme issue de secours que la situation parvint à se stabiliser sur la voie des taux d'intérêt décroissants. C'était là la phase cruciale, mieux connue de nos jours, de cette " Guerre des sept ans »(Aeschimann et Riché), une histoire des conflits autour du Franc fort et de la politique de désinflation compétitive. Mais c'est aussi la phase durant laquelle grandissait la résolution, tout particulièrement au sein du couple franco-allemand, de mettre en œuvre l'Union monétaire : de créer la monnaie commune.
Le basculement au DM fut un choc pour l'économie de l'ex-RDA

Elle consomme toujours plus qu'elle ne produit, et l'ouest finance son déficit courant

L'Unité fut aussi un grand choc pour le SME

La « Guerre des 7 ans » force l'idée d'une monnaie commune 


\section{L'Euro - et l'influence déterminante du discours franco-allemand}

Un adieu douloureux à toutes les monnaies nationales

Transfert de valeurs du DM à l'Euro
L'adieu au Deutsche Mark ne fut pas simple pour les Allemands, tout particulièrement ceux de l'est. Pour les Allemands de l'ouest, le Deutsche Mark symbolisait la renaissance d'une économie prospère. Pour les Allemands de l'est, il incarnait aussi l'expérience vécue d'une monnaie au fort pouvoir d'achat - et ce, dès avant l'Unité, via l'accès limité aux boutiques réservées aux achats en devises (les Intershops). Cela étant, l'adieu à leur monnaie ne fut pas simple non plus pour les autres membres de l'Union monétaire. Car l'argent, comme le souligne Kenneth Dyson, outre sa dimension technique et fonctionnelle, en a aussi une autre, politique et culturelle. Le Franc français ou la Livre britannique, qui connaissent une bien plus longue histoire que le deutsche Mark, "possèdent un pouvoir émotionnel et psychologique. Ils sont un moyen de conserver des valeurs, et pas seulement au sens économique, et d'ailleurs les pièces de monnaie représentent souvent des mythes nationaux savamment élaborés " (Dyson, 1994, p. 5). En ce sens, ces monnaies étaient à l'évidence fondatrices d'identité ; et la monnaie commune, l'Euro, "nourrit [maintenant à son tour] la conjuration d'une 'vision étatique' et d'une 'culture commune' ”.

$\mathrm{Si}$ au cours de sa brève histoire, le Deutsche Mark est devenu symbole, c'est que l'institution à l'arrière-plan remplissait assez bien son rôle technique et fonctionnel. C'est ainsi qu'Otmar Issing, principal maître d'œuvre de la conception de politique monétaire menée par la Banque Centrale Européenne, estime que deux éléments ont contribué à rendre l'adieu moins douloureux. Le premier était que « le statut de la BCE adopté à Maastricht s'inspire si étroitement du modèle de la Bundesbank qu'à lui seul, cet état de fait conforte la crédibilité de la promesse que l'Euro connaîtra la même stabilité que le Deutsche Mark" (Issing, 2008, p. 20). Le deuxième est que le Pacte de Stabilité et de Croissance avait valeur de garantie "contre les effets néfastes d'une politique de déficit budgétaire menée dans d'autres pays » (ibid.).

II semble donc bien que le Deutsche Mark, ou du moins sa structure institutionnelle, fête aujourd'hui ses 61 ans et se réjouisse d'une vigoureuse santé... (Soulignons au passage que l'allusion à la primauté de la monnaie sur la Loi fondamentale, telle qu'elle transparaît dans le titre de cette contribution, est bien entendu ironique et qu'elle se réfère à un cliché volontiers cultivé. La primauté va par nature à la Constitution, si ce n'est du point de vue chronologique, du moins par essence). La politique de stabilité poursuivie par une BCE soucieuse de son indépendance - et plus généralement par le Système européen des banques centrales, donc par chacune des banques centrales des Etats membres - est si incontestable que cette caractéristique apparaît même en filigrane dans les positions de ses plus féroces détracteurs. Le sociologue Pierre Bourdieu est même allé jusqu'à personnaliser sa critique de cette politique en évoquant le "système Tietmeyer ", tant il est vrai que Hans Tietmeyer, à l'époque président de la Bundesbank, incarne par son respect exemplaire de la doctrine le long cheminement dont est issu la monnaie européenne. Dans son ouvrage intitulé «Le défi de l'Euro » («Herausforderung Euro»), il décrit «la difficulté d'un processus préliminaire qui impliquait un choix conscient d'étapes intermédiaires et d'orientations, surtout dans les relations entre la France et l'Allemagne » (p.V).

Toujours selon Hans Tietmeyer, "sans [vouloir sous-estimer] la contribution d'autres Etats membres ou de la Commission ", la qualité de cette relation a été décisive tout au long de la longue genèse de l'Euro. C'est la raison pour laquelle il m'apparaît primordial que les discussions, trop souvent confinées dans les contextes nationaux respectifs, soient rendues accessibles au partenaire, grâce au travail d'institutions comme le CIRAC et de sa revue Regards sur l'économie allemande. Une lecture avertie et nuancée (ce qui n'était pas le propos de Bourdieu) de ces discussions met invariablement en évidence les diver- 
gences d'approche entre nos deux pays. Il apparaît ainsi par exemple que, en ce qui concerne l'efficacité du contre-pilotage macro-économique, le Conseil d'Analyse Economique défend depuis longtemps une autre position que le Conseil des experts pour l'examen de l'évolution économique globale (Sachverständigenrat zur Begutachtung der gesamtwirtschaftlichen Entwicklung), dont il est en partie l'homologue.

De même, on constate au sein de l'UEM des divergences sur la question de la nécessité d'une coordination des politiques budgétaires. Le point le plus litigieux étant alors la question d'un gouvernement économique, surtout lorsque celui-ci s'assimile à une coordination ex ante avec la politique monétaire. Rappelons ici cet épisode à première vue paradoxal qui s'était déroulé outre-Rhin en 1956. Pour ériger un système de défense contre les attaques dirigées contre l'indépendance de la Bank deutscher Länder, le ministre de l'Economie Ludwig Erhard avait pour ainsi dire "créé 'dans le dos' d'Adenauer [le] Conseil conjoncturel pour la coordination de la politique monétaire avec la politique budgétaire et la politique économique générale [Konjunkturrat zur Koordinierung der Geld- mit der Fiskal- und allgemeinen Wirtschaftspolitik] ». II était composé de représentants des ministères fédéraux de l'Economie et des Finances, ainsi que de la Bundesbank. Mais les décisions à proprement parler étaient prises par une commission composée de directeurs de service qui se réunissait une fois par mois. Elle débattait aussi au préalable les décisions de politique monétaire (voir Holtfrerich, 1998, pp. 394-395). Quelques décennies plus tard, en France, le ministre des Finances Dominique Strauss-Kahn proposait, en se fondant sur une argumentation similaire, la mise en œuvre d'une coordination institutionnelle au sein de l'UEM. D'elle naîtra l'Euro-Groupe.

Mais on Constate PAREILlement UNE LARGE CONVERGENCE de vues sur la plupart des thèmes cités ici à titre d'exemple. Et il semble que celle-ci se soit accrue au fil du temps. Cela n'apparaît bien sûr pas si on se contente de confronter les opinions médianes - ou pour le formuler d'une manière plus caricaturale : celle du Français et de l'Allemand. II convient toutefois de souligner à quel point les échanges franco-allemands, notamment entre les économistes, ont gagné en intensité au cours des dernières années. CAE et Conseil des Sages se réunissent régulièrement, le Cercle des économistes organise des séminaires conjoints avec des partenaires allemands. Quant aux économistes des banques ou des entreprises, ils se rencontrent depuis plus longtemps encore.

Tous ces échanges sont nécessaires parce que l'union monétaire est un arrangement institutionnel qui ne peut recueillir l'adhésion de tous qu'au prix d'un débat public permanent et sans tabou. Encore une fois, ce n'est pas parce qu'ils y auraient des prédispositions quasi-génétiques ou le vécu de deux chocs historiques que les Allemands sont devenus des adeptes de la stabilité. Les Français aussi, tout comme les Hollandais ou les Italiens, prisent la stabilité de leur pouvoir d'achat. Enfin, l'Histoire nous a enseigné quelles peuvent être les conséquences sociales désastreuses d'une monnaie instable (qu'on se rappelle l'impressionnante description qu'en a donnée Stefan Zweig). Voilà pourquoi les institutions qui garantissent une telle stabilité trouvent dans l'opinion une adhésion croissante. Voilà pourquoi aussi les banques centrales ont engagé un dialogue permanent avec l'espace public. La participation au débat public est une tradition judicieuse que poursuivent aujourd'hui les banques centrales des Etats membres comme la Banque centrale européenne. Car, pour en revenir aux propos de Ralf Dahrendorf, rendre compte de la sorte signifie non seulement œuvrer dans l'intérêt du citoyen, mais aussi dans celui d'une limitation du pouvoir, c'est-à-dire dans le sens de la liberté.

Traduction : I. Bourgeois

N.d.E. : Hans-Helmut Kotz s'exprime ici en son nom personnel
... surtout sur les divergences d'approche 


\section{Indications bibliographiques}

- Aeschimann Eric, Rıché Pascal, La guerre de sept ans. Histoire secrète du franc fort 1989-1996, Paris, Calman-Lévy, 1996

- Attal Jacques, Verbatim III, Paris, Fayard, 1996

- Blinder Alan, «The Rules-versus-Discretion Debate in the Light of Recent Experience », in Weltwirtschaftliches Archiv, 1987, pp. 399-413

- Buchнеiм Christoph, «Die Errichtung der Bank deutscher Länder und die Währungsreform in Westdeutschland », in Deutsche Bundesbank (ed), Fünfzig Jahre Deutsche Mark. Notenbank und Währung in Deutschland seit 1948, Munich, C.H.Beck, 1998, pp. 91-138

- Brociner Andrew, Chagny Odile, «La Bundesbank : une orthodoxie pragmatique », Revue de l'OFCE, vol. 56, n¹, 1996, pp. 95-119

- Coulbois Paul, « La politique monétaire en Allemagne depuis 1948 », Revue économique, Vol. 8 , n4, pp. 649 - 695

- Dyson Kenneth, Featherstone Kevin, The Road to Maastricht. Negociating Economic and Monetary Union, Oxford, OUP, 1999

- Dyson Kenneth, Elusive Union. The Process of Economic and Monetary Union in Europe, Londres, Longman, 1994

- Giersch Herbert, Paqué Karl-Heinz et Schmieding Holger, The Fading Miracle. Four Decades of Market Economy in Germany, Cambridge, Cambridge UP, 1994

- HANSMEYer Karl-Heinrich et CAESAR Rolf, «Kriegswirtschaft und Inflation (1936-1948) 》, in DeUtSChE Bundesbank (ed), Währung und Wirtschaft in Deutschland, Francfort, Fritz Knapp Verlag, 1976, pp. 367-429

- Holtfrerich Carl-Ludwig, «Geldpolitik bei festen Wechselkursen (1948-1970) », in DeUTSCHE Bundesbank (ed), Fünfzig Jahre Deutsche Mark. Notenbank und Währung in Deutschland seit 1948, Munich, C.H.Beck, 1998, pp. 347-438

- Issing Otmar, Der Euro. Geburt. Erfolg. Zukunft, Munich, Vahlen, 2008

- JAMES Harold, « Die Reichsbank 1876 bis 1945 », in DeUtsche BundesbaNk (ed), Fünfzig Jahre Deutsche Mark. Notenbank und Währung in Deutschland seit 1948, Munich, C.H.Beck, 1998, pp. 29-89

- Kotz Hans-Helmut, «À Francfort vers un assèchement des réserves d'épargne mondiales ? », in Revue d'Économie Financière, n¹5, 1990, pp. 227-231

- Kotz Hans-Helmut, «Quoi de neuf à la Buba ? », in Revue d’Économie Financière, n¹7, 1992, pp. 202-206

- Marsh David, The Bundesbank. The Bank that Rules Europe, London, Heinemann, 1992

- Marsh David, The Euro. The Politics of the New Global Currency, Yale:,Yale UP, 2009

- Mitzakis Michel, «La réforme monétaire en Allemagne occidentale ? ", in Revue économique, Vol. 1, N³, 1950, pp. 311-340

- MölleR Hans, « Die westdeutsche Währungsreform von 1948 », in DeUTSCHE BUNDESBANK (ed), Währung und Wirtschaft in Deutschland, Francfort, Fritz Knapp Verlag, 1976, pp. 433-483

- Neumann Manfred, « Geldwertstabilität: Bedrohung und Bewährung », in DEUTSCHE BuNDESBANK (ed), Fünfzig Jahre Deutsche Mark. Notenbank und Währung in Deutschland seit 1948, Munich, C.H.Beck, 1998, pp. 309-346

- Pierenkemper Toni, « La peur des Allemands devant l'inflation », in Rapport Moral, édition 1996

- Rıchter Rudolf, Deutsche Geldpolitik 1948-1998, Tübingen, Mohr Siebeck, 1999

- Schmidt Helmut, Die Deutschen und ihre Nachbarn. Menschen und Mächte II, Berlin, Siedler, 1990

- von HAGEN Jürgen, « Geldpolitik auf neuen Wegen », in DeUTSCHE Bundesbank (ed), Fünfzig Jahre Deutsche Mark. Notenbank und Währung in Deutschland seit 1948, Munich, C.H.Beck, 1998, pp. 439-473

- VorläNDER Hans, «Die Deutschen und ihre Verfassung », in Aus Politik und Zeitgeschichte, n¹8-19/2009, pp. 8-18

- WinckLer Heinrich August, Der lange Weg nach Westen. Deutsche Geschichte, vol. II, Munich, Beck, 2002. 